\title{
Guidance of cell adhesion and migration by graphitic nanopetals on carbon fibers
}

\author{
Soham Ghosh ${ }^{1}$, Guoping Xiong ${ }^{1,2}$, Timothy S. Fisher ${ }^{1,2}$, Bumsoo Han ${ }^{1,2,3,+}$
}

${ }^{1}$ School of Mechanical Engineering Department, Purdue University, West Lafayette, IN, USA

${ }^{2}$ Birck Nanotechnology Center, Purdue University, West Lafayette, IN, USA

${ }^{3}$ Weldon School of Biomedical Engineering, Purdue University, West Lafayette, IN, USA

${ }^{+}$Corresponding authors: Bumsoo Han, Ph.D.

585 Purdue Mall, West Lafayette, IN 47907, USA

email: bumsoo@purdue.edu 


\begin{abstract}
Carbon-based nanomaterials have emerged in a wide variety of applications because of their tunable mechanical, electrical, chemical and optical properties. However, poor cellular adhesion makes their use significantly limited in tissue engineering and regenerative medicines. In order to address this challenge, we studied interactions of fibroblast and epithelial cells with graphitic nanopetal structures grown on carbon fibers. We found that all the cell types studied attached to and migrated along the nanopetal coated carbon fibers, but not to the non-coated fibers. The petals' nanoscale irregular geometry is thought to enhance cellular adhesion and migration. Further understanding of the mechanisms of this interaction and improvising this method may be useful to design novel tissue engineering strategies using carbon-based nanomaterials.
\end{abstract}

Keywords: Nanopetal, Fibroblast, Epithelial Cells, Adhesion, Migration, Carbon-based Materials 


\section{Introduction}

Carbon-based nanomaterials such as carbon nanotubes/nanofibers [1,2], nanospheres [3], nanohorns [4] and nanopetals [5] have shown great promises to enable innovative materials because of their highly tunable mechanical, optical, chemical and electrical properties [6-10]. Two-dimensional (2D) structures in the form of single-layer graphene (SLG) or few-layer graphene (FLG) and three-dimensional graphitic nanostructures have found applications in electrochemical and biological sensors, energy storage, photovoltacic cells, optoelectronics, ultrafiltration and composite materials [11-13].

Despite the enormous promise and ease of modulation in their properties, interaction of carbon-based materials with biological cells remains challenging. Therefore though polymers [14], metals [15] and silicon based materials [16] have been successfully employed in tissue engineering and regenerative medicine, use of carbon-based materials in this context is only emerging. For example, limited success has been reported for cell culture in graphitic environments specific to neural cells and stem cells $[17,18]$. Interactions of cells with carbon nanotubes and graphitic sheets is known to be poor unless significant chemical modifications are performed, which can result in unwanted cytotoxicity. Studies have been performed with 2D graphitic structures to show their specific use in stem cell differentiation [19-22]. Some success is reported where neural stem cells interact with $3 \mathrm{D}$ graphitic foam but those porous structures were specifically coated for enhanced functionalization [23]. But generally many cell types do not adhere or respond to carbon based materials easily [24] unless they are modified chemically. Therefore, it is evident that new ways to enhance cell-material interactions are necessary to exploit the plethora of desirable properties of carbon-based materials in tissue engineering. 
In this paper, we report that biological cells strongly interact with, i.e., adhere to and migrate along, graphitic nanopetals (GNPs) grown on carbon fibers. This interaction is demonstrated in several cell types: dermal fibroblast, bronchial epithelial cells and breast carcinoma cells. Cell adhesion and migration on the graphitic structure are observed. The results are further expanded to study the modulation of the graphite nanostructural properties in tissue engineering and regenerative medicine applications.

\section{Materials and methods}

Carbon fibers coated with hydrogen terminated nanopetals (H-GNP) and oxygen terminated nanopetals (O-GNP) was created as described previously $[5,25,26]$ (see Supplementary information for details). To characterize the interaction of cells with GNP, response of fibroblast, IB3-1 and MCF-7 cells to H-GNP was investigated. Human dermal fibroblasts (BR5), human bronchial epithelial cells (IB3-1) and human breast carcinoma epithelial cells (MCF-7) were cultured (see Supplementary information for details). Cells were seeded on collagen gel polymerized with carbon fiber (bare/nanopetal coated). Response of cells to bare carbon fiber was used as control. The procedure is explained in Figure 1. Briefly, $2 \mathrm{~mL}$ collagen solution prepared from a high concentration type I rat tail collagen (BD Biosciences, Bedford, MA) so that the final collagen concentration was $3 \mathrm{mg} / \mathrm{mL}$ collagen. This was obtained by thorough mixing of 10\% 10X MEM, $30 \mathrm{mM}$ HEPES, $10 \mu \mathrm{g} / \mathrm{mL}$ penicillin/streptomycin, 2 $\mathrm{mM}$ L-glutamine, $6 \%$ fetal bovine serum, and $2.3 \%(\mathrm{v} / \mathrm{v}$ of collagen added) $1 \mathrm{~N}$ sodium hydroxide with the collagen. Then, distilled water was added to make a total volume of $2 \mathrm{~mL}$. The collagen solution was placed in a $48 \times 18 \mathrm{~mm}^{2}$ chamber slide (Lab-Tek II, Nunc, Naperville, IL) and allowed to partially polymerize at $37^{\circ} \mathrm{C}$ for $20 \mathrm{~min}$. At this stage, the isolated carbon fibers were placed on partially polymerized collagen solution and was let completely polymerize 
for 40 minutes. After polymerization, $2 \mathrm{~mL}$ of complete medium containing cells was added on top of the collagen gel. To understand the effect of termination on the cell-nanopetal interaction, response of fibroblast and IB3-1 cells to O-GNP was investigated.

Response of cells to carbon fibers (bare/GNP) was observed using an inverted microscope (Olympus, IX71) from 1 day after they were seeded. For time-lapse imaging to visualize dynamics of attachment and migration, cells were imaged every 30 seconds, while the chamber slide was placed in a stage-top incubator (Okolab, Italy) placed on the microscope. For detailed visualization of cell attachment on GNP covered carbon fibers, the cell membrane (Cellmask, Thermofisher) and the nucleus (Hoechst, Thermofisher) was stained at the end of time-lapse imaging.

\section{Results and discussion}

Figure 2 distinctly shows the morphological difference of the carbon fiber surface before and after GNP growth. The bare carbon fiber surface is relatively smooth with visible nanoscale grooves (left panel). However, after a one-step catalyst-free MPCVD growth process, GNPs consisting few layers of graphene (FLG) grow approximately 500 to $800 \mathrm{~nm}$ out from the carbon fiber surface, with a typical width of a single, unwrinkled 2D petal ranging from $100 \mathrm{~nm}$ to 900 $\mathrm{nm}$, and a petal thickness of a few nanometers. These GNPs uniformly cover the surface of carbon fibers, exhibiting distinct surface roughness (right panel). Note that the individual carbon fibers have a characteristic diameter of around 8-9 $\mu \mathrm{m}$.

Figure 3 shows how cells respond to the bare and GNP coated carbon fibers 1 day after they were seeded. All the cell lines, i.e., fibroblast, MCF-7 and IB3-1 show attachment to the HGNP-coated carbon fibers while the cells do not attach nor show any preferential alignment 
towards bare fibers (Figure 3a). Fibroblasts generally show a flat morphology on collagen gel. However, near the carbon fiber they show a slender morphology and the cell wraps around the fiber. MCF-7 shows rounded and clustered morphology on collagen gel typical of their nature but they also show a slender structure along the H-GNP carbon fiber on which they are attached. Similar behavior occurs in IB3-1 cells on H-GNP carbon fiber. In Figure 3b, bare and GNP grown fibers are shown together. It is clearly apparent that fibroblasts align and wrap around the fibers while cells near bare fibers show no preferential alignment/ attachment. Figure $3 \mathrm{c}$ shows a fibroblast (green) wrapping around a H-GNP carbon fiber; the nucleus is shown in blue. Cells also attach on oxygen functionalized graphitic nanopetals (O-GNP) as shown in Figure 3d, without any visible morphological difference than that of H-GNP.

The dynamics of cell attachment on the GNP and subsequent cell migration are presented in Figure 4. As observed from Figure 4a (also see Supplementary video 1), fibroblasts initiate the interaction with the GNP coated carbon fiber nearby, and spread along the fiber within one hour after the cells are seeded. Note three such cells in the field of view with one pointed with arrowheads. Interestingly these attached cells migrate along the fiber as observed in Figure $4 \mathrm{~b}$ (also see Supplementary video 2) after 1 day of cell seeding. For fibroblasts, this migration is observed from right after attachment till three days after seeding as long as the collagen construct is intact and cells are viable. Depending on cell type and initial concentration of cells, the time span of collagen construct integrity and cell viability varied. The specific arrow-pointed cell in the field of view migrates approximately 70 microns along the fiber in 30 minutes while other cells in the same field of view remains stationary on collagen gel. Generally fibroblasts showed better migration behavior, but it was not quantified. Further investigation to quantify the cell specific migration behavior is warranted. 
These results suggest that all cell types studied, fibroblast and epithelial, preferentially interact with the GNPs. Since the chemical residue on carbon fiber is degraded during the GNP formation process [5], chemical treatment is not thought to be a primary reason for cellular attachment, as confirmed further by the fibroblast cells attaching on GNP coated carbon fiber but not on the bare one as shown in Figure $3 b$.

Preferential functionalization of nanopetals with oxygen and hydrogen does not show any effect on cell adhesion behavior, though other functionalization was not attempted. Therefore the interaction is most likely driven by mechanical irregularities. Two major mechanisms have been proposed to explain cellular adhesion to their surroundings. First, biological cells adhere with their surroundings using numerous adhesion proteins e.g., integrin, vinculin, paxilin, and cadherin [27-29]. This interaction is mediated by molecular scale chemical bonding that normally occurs in artificial or native tissues. Secondly, at a larger scale, cells may interact with its environment using larger surfaces and grooves with cell-specific dimensions as demonstrated with several non-carbon based substrate materials [30-35]. The interaction observed in the present study is thought to be similar to the later and may be caused by the cell blebbing mechanism [36,37] that exploits the irregularities created by petals. Cells may use such physical irregularities to mechanically attach through the grooves. The bleb formation in cells has a characteristic scale of tens to hundreds of nanometer [38], which reasonably matches with the scale of graphitic nanopetal structure irregularities (Figure 4c). It is highly likely that those bleb formations mechanically interact with the GNP irregularities by a latch and release mechanism.

Substrate stiffness is known to cause significant alteration in cell adhesion $[39,40]$. Stiffness of individual nanopetal measured as $10^{4}-10^{5} \mathrm{~Pa}$ [41] and surface roughness of the nanopetals, which is approximately $500 \mathrm{~nm}$ [42], may affect the cell adhesion. The petal size can 
be regulated by the plasma power, thus the surface roughness can be modified as required. The resulting cell adhesion behavior can be different based on the available nanopetal surface area [43].

Interaction of biological cells with carbon based materials is limited and this research advances a possible strategy to overcome this limitation. Although further research is warranted to validate the detailed interaction mechanisms, the present observation implies that graphitic nanopetals can be used to guide cell adhesion and migration on carbon-based materials for tissue engineering and regenerative medicine.

\section{Conclusion}

Graphitic nanopetals can significantly enhance the cellular adhesion of fibroblasts and epitheial cells on carbon fibers. Termination of the nanopetals with hydrogen and oxygen did not significantly alter the attachment behavior. After the adhesion, fibroblasts migrate along the graphitic nanopetal coated carbon fibers. This observation, upon further investigation on the detailed cell-nanopetal interaction mechanisms, may be utilized to guide cellular behavior on carbon based materials for tissue engineering and regenerative medicine.

\section{Acknowledgement}

We acknowledge the help provided by Seungman Park and Taylor J Thompson in the cell culture procedure and Anurag Kumar in the GNP carbon fiber preparation procedure. This research was partially supported by U.S. AFRL, AFOSR (FA9550-12-1-0037) under MURI Program (T.S.F.), U.S. National Science Foundation (1344654) (T.S.F.) and a Research Incentive Grant from Purdue University (B.H.).

\section{Conflict of interest}


The authors do not have any conflict of interest. 


\section{FIGURES}

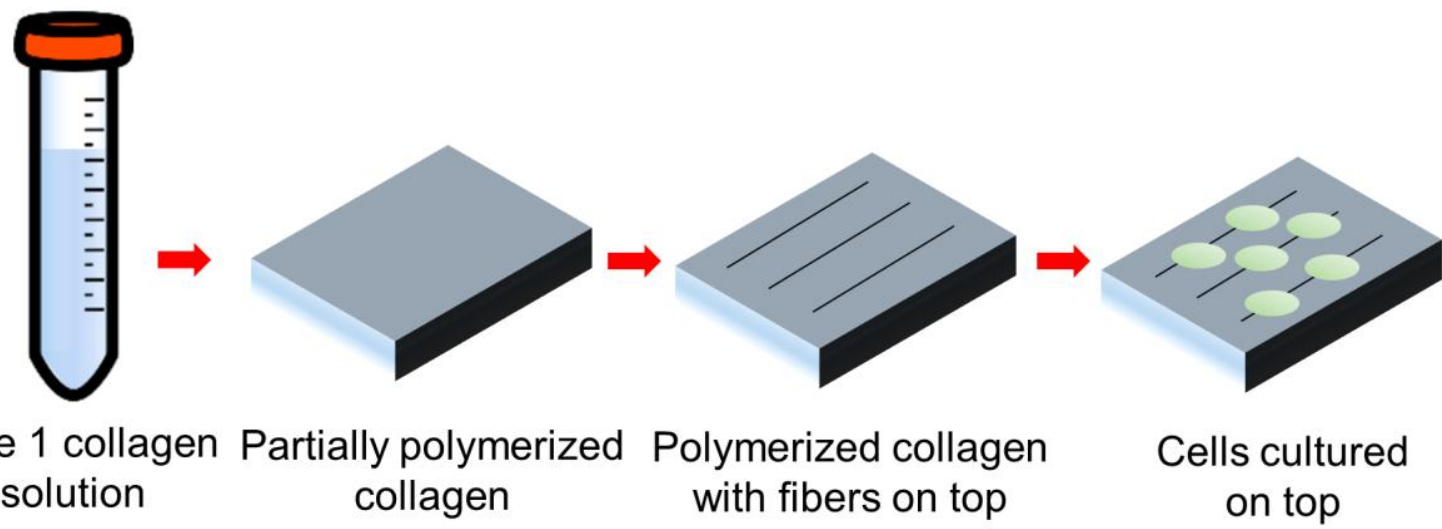

Figure 1. Schematic of cell-carbon fiber interaction experimental set up. Type 1 collagen solutions are polymerized. While partially polymerized, individual carbon fibers were embedded on top of collagen gel. After complete polymerization, cells were cultured on top of carbon fiber and polymerized collagen gel. 

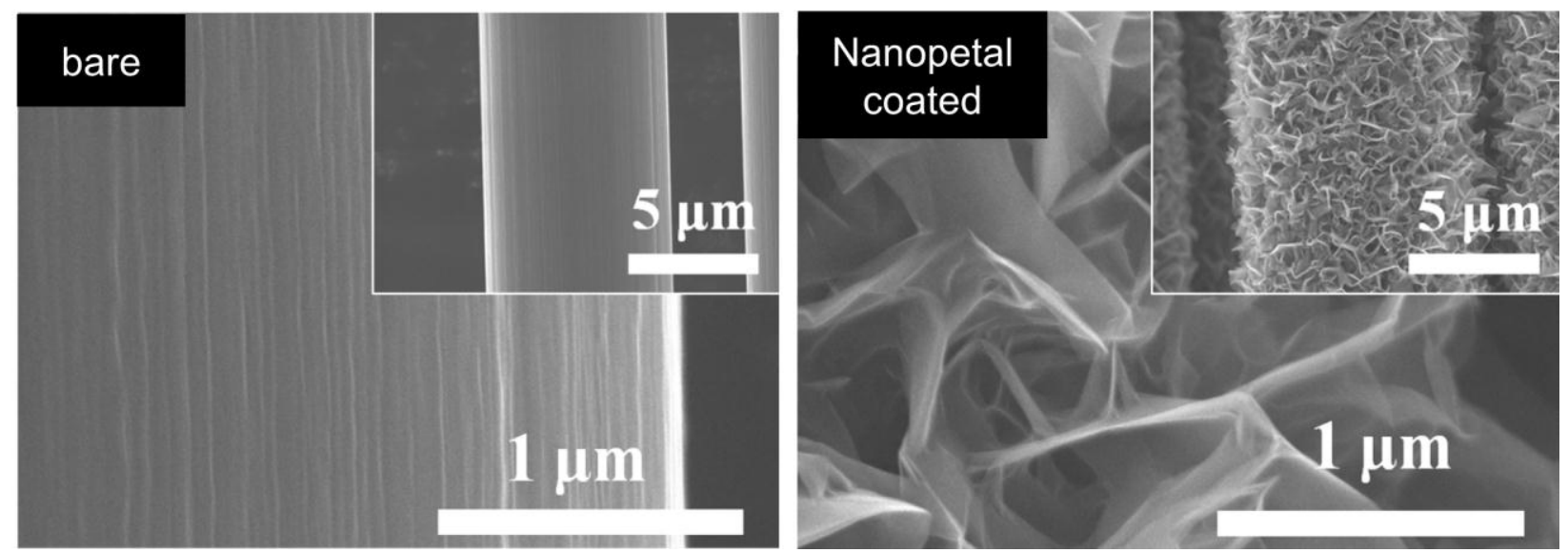

Figure 2. SEM images of the bare carbon fiber (left) and graphitic nanopetal (NP) grown on carbon fiber (right). 
(a)
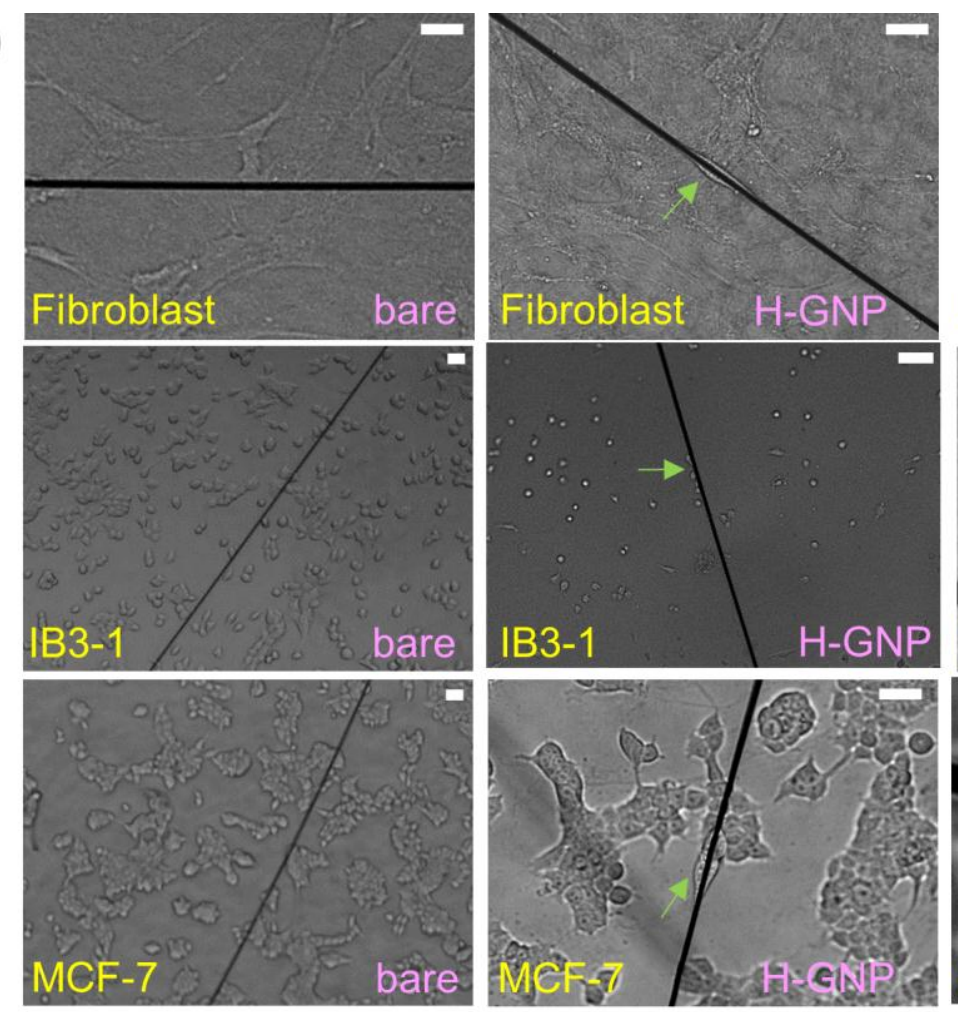

(b)

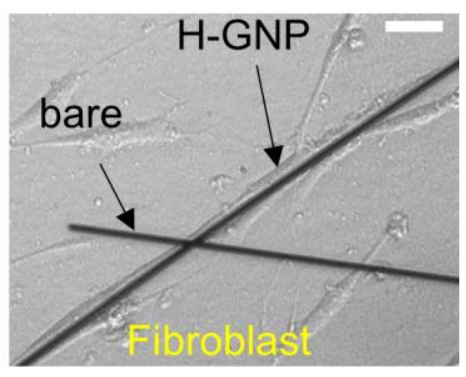

(c)

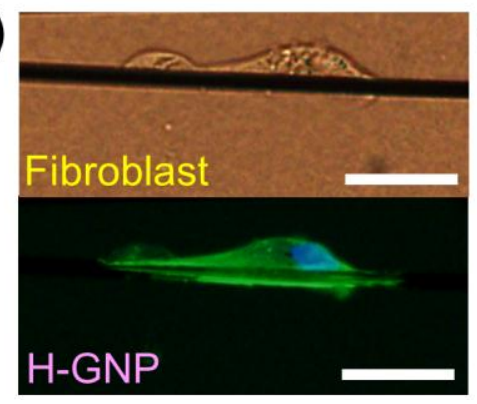

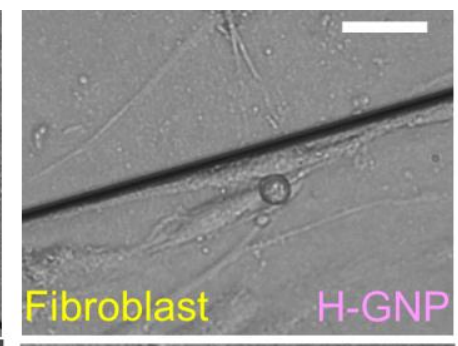
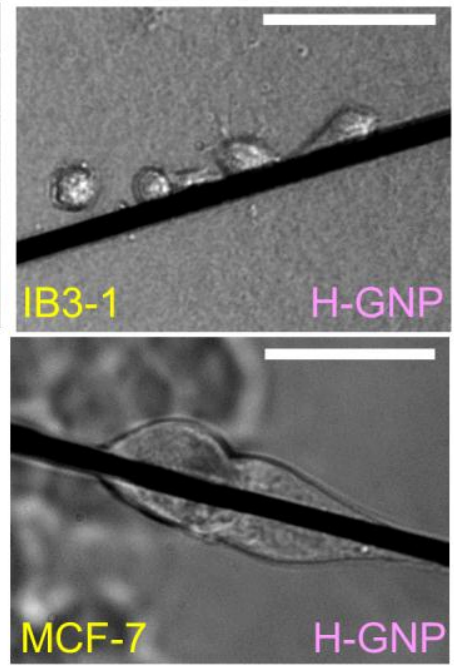

(d)

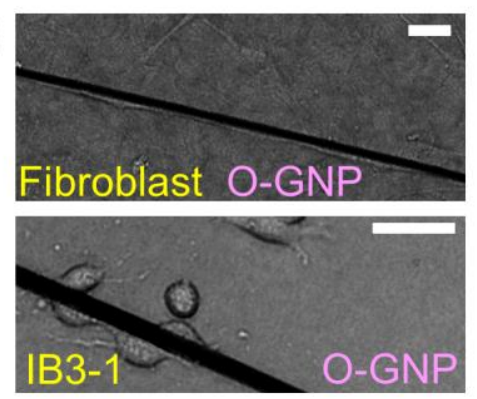

Figure 3. (a) Post 1 day response of fibroblast, IB3-1 and MCF-7 cells to bare carbon fiber (left column), to H-GNP fiber (middle column) and to H-GNP fiber, imaged at high magnification (right column). Cells interacting with NP coated fibers are shown with arrowheads. H-GNP fiber is carbon fiber with H-terminated graphitic nanopetal grown on it. (b) Fibroblast in same field of view with bare and H-GNP fiber. (c) Cell contour (green) and nucleus (blue) of a fibroblast wrapping a H-GNP fiber. (d) Post 1 day response of fibroblast and IB3-1 cells to O-GNP fiber. (Scale bar: $40 \mu \mathrm{m}$ ). 
(a)
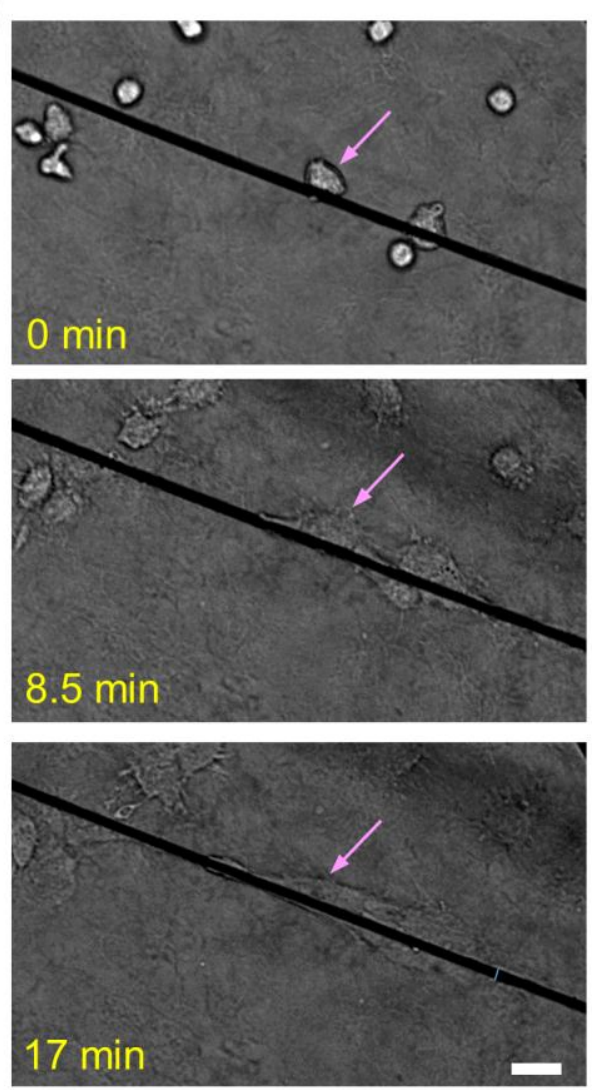

(b)
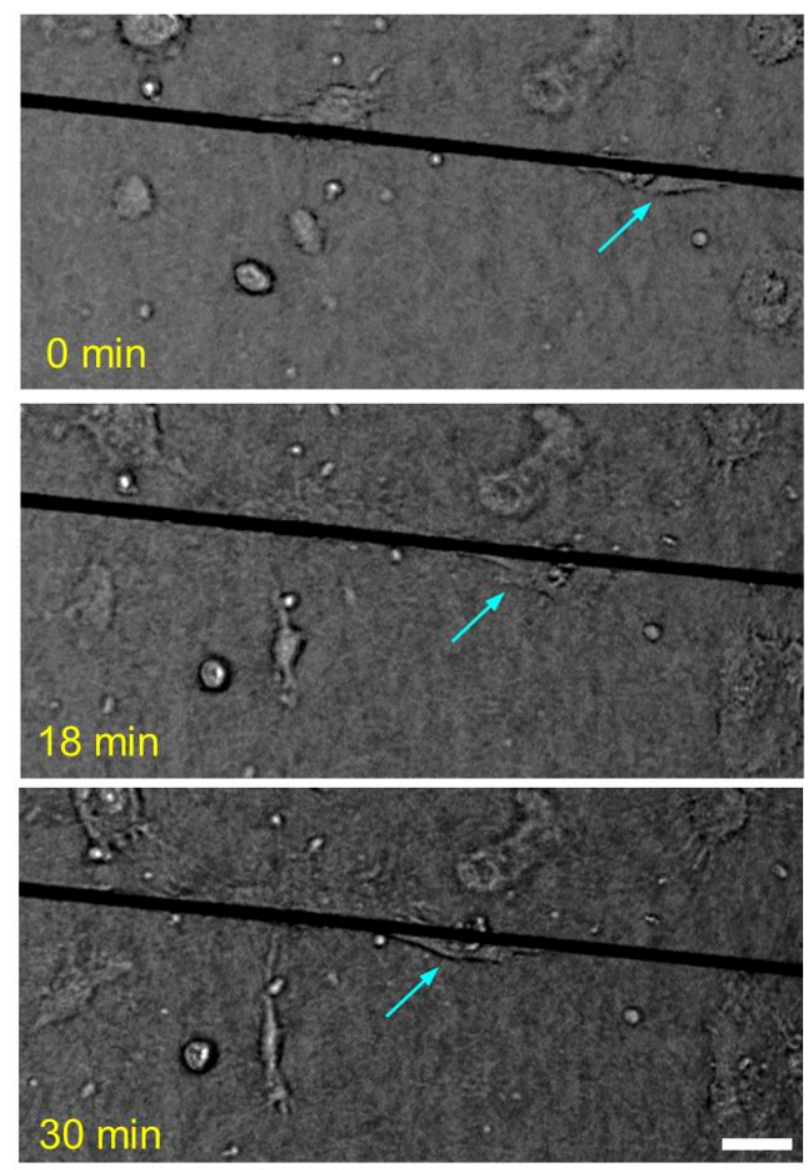

(c)

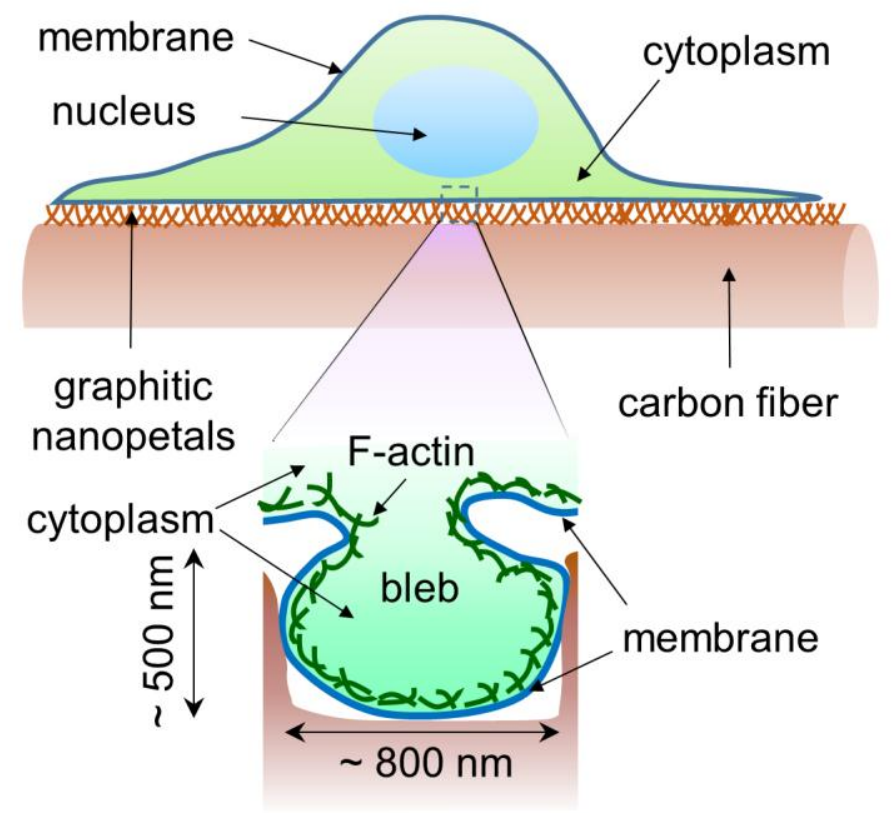


Figure 4. (a) Fibroblast attachment dynamics on H-GNP fiber after they are seeded (see supplementary video 1). (b) Migration of fibroblast along H-GNP fiber (see supplementary video 2). (Scale bar: $40 \mu \mathrm{m}$ ). (c) Schematic of proposed interaction mechanisms of cell with graphitic nanopetals. The petals provide nanoscale features which matches with the spatial scale of bleb formation. Thus cells can preferentially interact with petals, but not with carbon fibers. Dynamics of several accessory molecules and F-actin reorganization is responsible for creation and retraction of blebs, thus leading to a possible 'latch and release' mechanism [36-38]. 


\section{References}

[1] S. Iijima, Nature. 354 (1991) 56-58.

[2] P. He, L. Liu, W. Song, G. Xiong, T.S. Fisher, T. Chen, RSC Adv. 5 (2015) 3183731844.

[3] B.R. Selvi, D. Jagadeesan, B.S. Suma, G. Nagashankar, M. Arif, K. Balasubramanyam, M. Eswaramoorthy, T.K. Kundu, Nano Lett. 8 (2008) 3182-3188.

[4] A. Krishnan, E. Dujardin, M.M.J. Treacy, J. Hugdahl, S. Lynum, T.W. Ebbesen, Nature. 388 (1997) 451-454.

[5] G. Xiong, K.P.S.S. Hembram, D.N. Zakharov, R.G. Reifenberger, T.S. Fisher, Diam. Relat. Mater. 27-28 (2012) 1-9.

[6] K. S. Novoselov; A. K. Geim; D. Jiang; Y. Zhang; S. V. Dubonos; I. V. Grigorieva; A. A. Firsov, Science. 306 (2004) 666-669.

[7] K.S. Novoselov, A.K. Geim, S. V Morozov, D. Jiang, M.I. Katsnelson, I. V Grigorieva, S. V Dubonos, A.A. Firsov, Nature. 438 (2005) 197-200.

[8] G. Xiong, C. Meng, R.G. Reifenberger, P.P. Irazoqui, T.S. Fisher, Electroanalysis. 26 (2014) 30-51.

[9] X. Xu, H. Li, Q. Zhang, H. Hu, Z. Zhao, J. Li, J. Li, Y. Qiao, ACS Nano. 9 (2015) 39693977.

[10] Q. Zhang, X. Xu, H. Li, G. Xiong, H. Hu, T.S. Fisher, Carbon. 93 (2015) 659-670.

[11] F. Bonaccorso, Z. Sun, T. Hasan, A.C. Ferrari, Nat. Photonics. 4 (2010) 611-622.

[12] K.S. Novoselov, V.I. Fal'ko, L. Colombo, P.R. Gellert, M.G. Schwab, K. Kim, Nature. 490 (2012) 192-200.

[13] Y. Shao, J. Wang, H. Wu, J. Liu, I.A. Aksay, Y. Lin, Electroanalysis. 22 (2010) 10271036.

[14] D.S. Kohane, R. Langer, Polymeric biomaterials in tissue engineering, Pediatr. Res. 63 (2008) 487-491.

[15] S. Bose, M. Roy, A. Bandyopadhyay, Trends Biotechnol. 30 (2012) 546-554.

[16] J.L. Coffer, M.A. Whitehead, D.K. Nagesha, P. Mukherjee, G. Akkaraju, M. Totolici, R.S. Saffie, L.T. Canham, Phys. Status Solidi Appl. Mater. Sci. 202 (2005) 1451-1455.

[17] G.Y. Chen, D.W.P. Pang, S.M. Hwang, H.Y. Tuan, Y.C. Hu, Biomaterials. 33 (2012) 418-427.

[18] S. Ryu, B.-S. Kim, Tissue Eng. Regen. Med. 10 (2013) 39-46.

[19] V. Lovat, D. Pantarotto, L. Lagostena, B. Cacciari, M. Grandolfo, M. Righi, G. Spalluto, M. Prato, L. Ballerini, Nano Lett. 5 (2005) 1107-1110. 
[20] T.R. Nayak, H. Andersen, V.S. Makam, C. Khaw, S. Bae, X. Xu, P.L.R. Ee, J.H. Ahn, B.H. Hong, G. Pastorin, B. Ozyilmaz, ACS Nano. 5 (2011) 4670-4678.

[21] S.Y. Park, J. Park, S.H. Sim, M.G. Sung, K.S. Kim, B.H. Hong, S. Hong, Adv. Mater. 23 (2011) 263-267.

[22] Y. Wang, W.C. Lee, K.K. Manga, P.K. Ang, J. Lu, Y.P. Liu, C.T. Lim, K.P. Loh, Adv. Mater. 24 (2012) 4285-4290.

[23] N. Li, Q. Zhang, S. Gao, Q. Song, R. Huang, L. Wang, L. Liu, J. Dai, M. Tang, G. Cheng, Sci. Rep. 3 (2013) 1604.

[24] H. Stoll, I.K. Kwon, J.Y. Lim, Neural Regen. Res. 9 (2014) 1810-1813.

[25] G. Xiong, K.P.S.S. Hembram, R.G. Reifenberger, T.S. Fisher, J. Power Sources. 227 (2013) 254-259.

[26] G. Xiong, C. Meng, R.G. Reifenberger, P.P. Irazoqui, T.S. Fisher, Adv. Energy Mater. 4 (2014) 1-9.

[27] M.R. Morgan, M.J. Humphries, M.D. Bass, Nat. Rev. Mol. Cell Biol. 8 (2007) 957-69.

[28] J.D. Hood, D.A. Cheresh, Nat. Rev. Cancer. 2 (2002) 91-100.

[29] J.T. Parsons, A.R. Horwitz, M. a Schwartz, Nat. Rev. Mol. Cell Biol. 11 (2010) 633-43.

[30] L. Cai, L. Zhang, J. Dong, S. Wang, Langmuir. 28 (2012) 12557-12568.

[31] M.G. Henry, L. Cai, X. Liu, L. Zhang, J. Dong, L. Chen, Z. Wang, S. Wang, Langmuir. 31 (2015) 2851-2860.

[32] H. Gao, H. Dong, X. Cao, X. Fu, Y. Zhu, C. Mao, Y. Wang, Langmuir. 31 (2015) 67976806.

[33] I. Tonazzini, E. Jacchetti, S. Meucci, F. Beltram, M. Cecchini, Adv. Healthc. Mater. 4 (2015) 1849-1860.

[34] K. Franze, P. a. Janmey, J. Guck, Annu. Rev. Biomed. Eng. 15 (2013) 227-51.

[35] Z. Li, Y. Gong, S. Sun, Y. Du, D. Lü, X. Liu, M. Long, Biomaterials. 34 (2013) 76167625 .

[36] E.K. Paluch, E. Raz, Curr. Opin. Cell Biol. 25 (2013) 582-590.

[37] G. Charras, E. Paluch, Nat. Rev. Mol. Cell Biol. 9 (2008) 730-736.

[38] O.T. Fackler, R. Grosse, J. Cell Biol. 181 (2008) 879-884.

[39] J. Huang, X. Peng, C. Xiong, J. Fang, J. Colloid Interface Sci. 355 (2011) 503-508.

[40] T. Yeung, P.C. Georges, L.A. Flanagan, B. Marg, M. Ortiz, M. Funaki, N. Zahir, W. Ming, V. Weaver, P.A. Janmey, Cell Motil. Cytoskeleton. 60 (2005) 24-34.

[41] A. Kumar, M.R. Maschmann, T.S. Fisher, S.L. Hodson, Carbon. 84 (2014) 236-245.

[42] K. Shiji, M. Hiramatsu, A. Enomoto, M. Nakamura, H. Amano, M. Hori, Diam. Relat. 
Mater. 14 (2005) 831-834.

[43] N.D. Gallant, K.E. Michael, A. J. Garcia, Mol. Biol. Cell. 16 (2005) 4329-4340. 